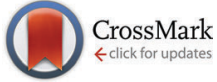

Cite this: Phys. Chem. Chem. Phys., 2015, 17, 17967

Received 20th April 2015 Accepted 15th June 2015 DOI: $10.1039 / c 5 c p 02294 k$

www.rsc.org/pccp

\title{
High-throughput approach for the identification of anilinium-based ionic liquids that are suitable for electropolymerisation $\dagger$
}

\author{
Muhammad E. Abdelhamid, ${ }^{\text {ab }}$ Timothy Murdoch, ${ }^{c}$ Tamar L. Greaves, ${ }^{\text {ac }}$ \\ Anthony P. O'Mullane ${ }^{\star d}$ and Graeme A. Snook*b
}

\begin{abstract}
We report the synthesis of new protic ionic liquids (PILs) based on aniline derivatives and the use of highthroughput $(\mathrm{HT})$ techniques to screen possible candidates. In this work, a simple HT method was applied to rapidly screen different aniline derivatives against different acids in order to identify possible combinations that produce PILs. This was followed by repeating the HT process with a Chemspeed robotic synthesis platform for more accurate results. One of the successful combinations were then chosen to be synthesised on a larger scale for further analysis. The new PILs are of interest to the fields of ionic liquids, energy storage and especially, conducting polymers as they serve as solvents, electrolytes and monomers at the same time for possible electropolymerisation (i.e. a self-contained polymer precursor).
\end{abstract}

\section{Introduction}

The polymerisation of aniline into PANi can be achieved via chemical or electrochemical oxidative polymerisation. ${ }^{1-3}$ The resulting conductive polymer films can be applied as supercapacitors, actuators or as photovoltaic devices. ${ }^{1,4}$ However a significant consideration to achieve both high quality films and high polymerisation yields is the provision of an excess of protons within the polymerisation medium. ${ }^{2,5}$ The electropolymerisation of aniline in some ionic liquids (ILs) results in polyaniline (PANi) films with unique properties. This is because PANi grows in ILs in a different way than in molecular and organic solvents. ${ }^{6}$ A broad range of ionic liquids (ILs) have been used in many applications in electrochemistry and are attractive because they can serve as solvents as well as electrolytes. ${ }^{7,8}$ ILs have been utilised as polymerisation media for polyaniline

\footnotetext{
${ }^{a}$ School of Applied Sciences, RMIT University, GPO Box 2476, Melbourne, VIC 3001, Australia

${ }^{b}$ Mineral Resources, Commonwealth Science and Industrial Research Organisation (CSIRO), Private Bag 10, Clayton, VIC 3169, Australia.

E-mail: Graeme.Snook@csiro.au

${ }^{c}$ Manufacturing, Commonwealth Science and Industrial Research Organisation (CSIRO), Private Bag 10, Clayton, VIC 3169, Australia

${ }^{d}$ School of Chemistry, Physics and Mechanical Engineering, Queensland University of Technology, GPO Box 2434, Brisbane, Qld 4001, Australia.

E-mail: anthony.omullane@qut.edu.au

$\dagger$ Electronic supplementary information (ESI) available: NMR spectra, viscosity measurements and density measurements of $n$-methylanilinium TFA, $n$-methylanilinium sulphate, $n$-ethylanilinium sulphate, and $n$-ethylanilinium methanesulfonate. See DOI: 10.1039/c5cp02294k
}

in many ways such as in mixtures of ILs with molecular solvents or acids. ${ }^{9-11}$

The search for the optimal IL for a specific application, such as the electropolymerisation of aniline, can be difficult and time consuming, as ILs are composed of cations and anions, of which there are a vast number of potential combinations. Furthermore, the incorporation of molecular or organic solvents increases the sample size dramatically due to the vast number of possible solvents and concentration ratios. However, highthroughput (HT) techniques, which have become widely acceptable for the rapid study and identification of new drugs and materials, can be applied. ${ }^{12-14}$ The technique involves cross screening and analysis of a large variety of starting materials ultimately leading to the identification of promising combinations. ${ }^{15}$ Such, HT techniques would therefore in principle be useful in IL synthesis as it would allow for the rapid screening and identification of potential ILs that are suitable for specific applications.

A particularly interesting subset of ILs, called protic ionic liquids (PILs) have the potential to be highly effective for the electropolymerisation of polymers that require the presence of protons. They are produced via proton transfer from a Brønsted acid to a Brønsted base. ${ }^{16,17}$ They are cheaper and easier to synthesise than aprotic ILs as they require only simple equimolar acid-base reactions. ${ }^{18}$ This, along with their generally lower viscosity compared with aprotic ionic liquids, ${ }^{16}$ makes them highly suitable for the high-throughput synthesis technique as demonstrated by Greaves et al. ${ }^{16}$ In addition, aniline and its derivatives are suitable candidates for a cationic precursor for PILs as they can act like Brønsted bases as shown by recent attempts. ${ }^{19}$ 
In this paper, a stoichiometric ratio of various combinations of aniline and aniline derivatives (i.e. as bases) with various acids were cross screened in a high throughput manner to short list the most promising anilinium-based protic ionic liquids (PILs), which were then further characterised. A few PILs were identified and the optimum combination of $\mathrm{N}$-ethylanilinium and TFA was chosen for full scale synthesis and characterisation. The new PIL was synthesised via a previous method used by Snook et $a l .{ }^{4}$ The benefit of synthesising such PILs is to utilise them for the direct production of conducting polyaniline derivatives without the addition of further solvents or proton donors, such as acids. ${ }^{2}$ They are a self-contained polymerisation system as they act as a polymerisation medium "solvent and electrolyte", and pre-protonated monomer at the same time, which can in principle be polymerised into the polyaniline derivative. This method is a greener approach to polymerise anilines as it reduces waste by eliminating the need to use other solvents, and removes the acid protonation step required during conventional polymerisation of aniline. Furthermore, this highly novel approach results in new morphologies of conducting polymers offering advanced properties of these materials.

\section{Experimental section}

\section{Materials}

$N$-Ethylaniline, $n$-methylaniline, 2-ethyl-6-methylaniline, 2,5dimethylaniline, 2,6-dimethylaniline, 3,5-dimethylaniline, 2-ethylaniline, 3-ethylaniline (Sigma Aldrich), trifluoroacetic acid (Sigma Aldrich, TFA), hydrochloric acid (Merck Millipore, 65\% $\mathrm{HNO}_{3}$ ), formic acid (Sigma-Aldrich), nitric acid (Merck Millipore, 37\% $\mathrm{HCl}$ ), sulphuric acid (Ajax Finechem Pty Ltd, 98\% $\mathrm{H}_{2} \mathrm{SO}_{4}$ ) and methanesulfonic acid (Sigma-Aldrich, 99.5\% $\mathrm{CH}_{3} \mathrm{SO}_{3} \mathrm{H}$ ) were used as received. In addition, pentane, absolute ethanol (Merck Millipore, EMSURE ACS), diethyl ether (Sigma-Aldrich) and 18.2 $\mathrm{M} \Omega \mathrm{cm}$ Milli-Q water were also utilised.

\section{Synthesis methods}

In order to screen the various acids against the aniline derivatives, two high-throughput methods were used. The first method is the simplest as a predetermined volume of the derivatives were loaded manually into Eppendorf tubes followed by the addition of the acids to start the reaction. This method is a quick and simple way to get preliminary screen data suitable for a large number of reactants.

The second method is an automated process where the loading of samples and the reactions are controlled by a robotic platform. This method is more accurate than the Eppendorf method as the reaction conditions, such as temperature and reaction time, are easily controlled and stoichiometric proportions are used of the acids and bases.

Following the two screening techniques, one promising acid-base combination was chosen to be synthesised on a larger scale.
Eppendorf tube high-throughput screen. In this method, a few drops of aniline and its derivatives were loaded into seven separate Eppendorf tubes. Subsequently, an approximately

Table 1 Illustrates the different acids and aniline derivatives that were screened via two high-throughput methods and their products

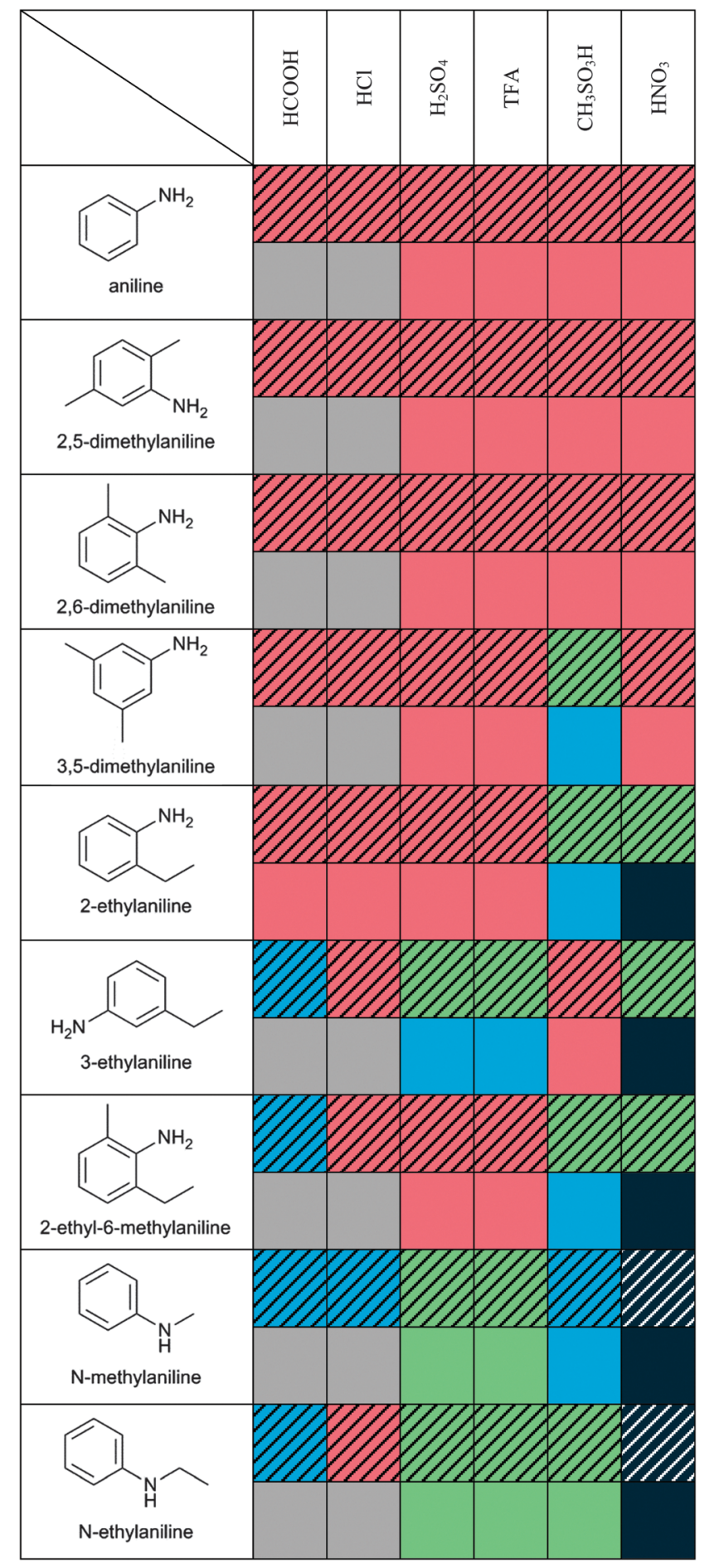

Solid, liquid (not IL), dark (oxidised) liquid, and possible PIL (patterned cells are for the test tube high-throughput and solid cells are for the Chemspeed robotic synthesis). 
equimolar amount of acid was added drop-wise to each derivative while stirring (Table 1 ).

Chemspeed robotic synthesis platform. The Chemspeed robotic synthesis platform contained an ISYNTH reactor block equipped with an array of 24 vials of $20 \mathrm{~mL}$ volume. The IL synthesis protocol was designed to mimic the conventional synthesis of protic ionic liquids where ethanol was added to aniline and its derivative solutions in order to ensure a liquid state during addition of the acid. The concentrations of the aniline (derivatives) in ethanol stock solutions were $1.0 \mathrm{M}$ as well as for the acids stock solutions.

The robotic platform dispenses liquids by means of an articulated 4-needle tool connected to four individual syringe pumps. This allows simultaneous aspiration/dispensing of aniline (derivatives) or acids to/from four adjacent reactor vials. Each syringe pump can operate individually to deliver different volumes, and at different rates to the other syringes. In this particular application, the platform was fitted with four $10 \mathrm{~mL}$ syringe pumps, and four stainless steel needles (ID $=0.8 \mathrm{~mm}$ ). The synthesis was conducted in two sequential batches of 24 PIL mixtures. The basic experimental steps programmed into the Chemspeed workflow for each batch are described below.

(1) Reagent bases (aniline derivatives + ethanol) and acids were loaded into $60 \mathrm{~mL}$ reservoir vials and fitted with purge septa.

(2) ISYNTH reactors were configured to be open under inert atmosphere (to prevent condensation) and cooled to a target temperature of $-10{ }^{\circ} \mathrm{C}$.

(3) Each of the six aniline derivative + ethanol mixtures were sequentially dispensed to four ISYNTH reactor vials such that all 24 reactor vials contain the aniline derivative. They were

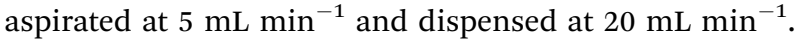

(4) Agitation/shaking of reactors was turned on (400 RPM).

(5) Each of the four acids were then added drop-wise to four ISYNTH reactors such that all 24 reactor vials contained acid + base. Acids were aspirated at $10 \mathrm{~mL} \min ^{-1}$ and dispensed at $0.1 \mathrm{~mL} \min ^{-1}$ to achieve drop-wise addition over an extended period of time.

(6) The reactors were agitated for an additional hour following the final acid addition. Due to the drop-wise addition of acid, all reactions had sufficient time to react between 2 and 6 hours.

(7) ISYNTH reactors were configured to be closed. Vacuum pressure in the reactors was stepped down gradually to a target pressure of $1 \mathrm{mbar}$ on the attached vacuum pump. At the same time the temperature was set to $38{ }^{\circ} \mathrm{C}\left(43{ }^{\circ} \mathrm{C}\right.$ on attached cryostat).

(8) Vacuum, heat, and agitation were continued overnight, although a review of the $\log$ data indicates that minimal pressure was obtained within 2 hours of starting step 7 .

(9) Pressure and temperature were returned to ambient conditions before the 24 reactor vials were removed, capped and taken away for analysis.

(10) Steps 1-9 were repeated for batch 2, which used the same three remaining bases, and the four acids.

Synthesis of $n$-ethylanilinium trifluoroacetate. A larger quantity of $n$-ethylanilinium TFA was prepared by reacting equimolar amounts of $n$-ethylaniline and trifluoroacetic acid (TFA). 0.1 mole of $n$-ethylaniline was added into a round-bottom flask with $5 \mathrm{~mL}$ of absolute ethanol. Connected to the roundbottom flask was a titration column loaded with 0.1 mole of TFA, $3 \mathrm{~mL}$ of absolute ethanol and $2 \mathrm{~mL}$ of milli-Q water. The acid mixture was added drop-wise to the $n$-ethylaniline solution under stirring and controlled temperature under $-10{ }^{\circ} \mathrm{C}$ in order to prevent the oxidation of the amine into an amide. After the reaction was finished, a yellow-orange viscous liquid was yielded in the flask. The product was dried under dynamic vacuum at $35{ }^{\circ} \mathrm{C}$ for 2 hours and then kept under static vacuum overnight at room temperature.

The dried product was a yellow to brown slushy solid with a melting point of approximately $42{ }^{\circ} \mathrm{C}$. The solid was purified through dissolving it in pentane and then adding ethyl ether to crash out the product as a white solid. The white solid was collected and dried via the rotary evaporator at $35{ }^{\circ} \mathrm{C}$ for an hour. The water content of the dried solid was measured to be 15 ppm. To obtain a liquid IL solution at room temperature, $150 \mu \mathrm{L}$ of milli-Q water was added to $1 \mathrm{~g}$ of the dried white solid and the mixture was heated to about $50{ }^{\circ} \mathrm{C}$ with a heat gun. This resulted in a light yellow-orange viscous liquid at room temperature with a measured water content of $162 \mathrm{ppm}$.

Characterisation. All the acid-base mixtures produced by the Chemspeed robotic platform and the individually synthesised $n$-ethylanilinium TFA were characterised by ${ }^{1} \mathrm{H}$ NMR and ${ }^{13} \mathrm{C}$ NMR using Bruker BioSpin Av400X NMR Spectrometers in order to confirm the proton transfer from the acid to the base and the structure of the products. Furthermore, conductivity measurement of the ILs was undertaken by SevenMulti dual $\mathrm{pH}$ and conductivity meter (Mettler Toledo), the density and viscosity were measured by Anton Paar density meter with External Measuring Cell and Anton Paar Rolling-ball viscometer Lovis 2000 in combination with A\&D SV-1A tuning fork vibro viscometers respectively.

\section{Results}

Fig. 1, shows $n$-ethylaniline and its products after the addition of different acids. Four possible outcomes were considered: (1) a solid forms, (2) non-ionic liquid forms (no proton transfer), (3) spontaneous polymerisation occurs (dark solid) or (4) the desired outcome of a protic ionic liquid forms. The combinations that resulted in potential ILs were then synthesised using the Chemspeed robotic platform in a high-throughput manner,

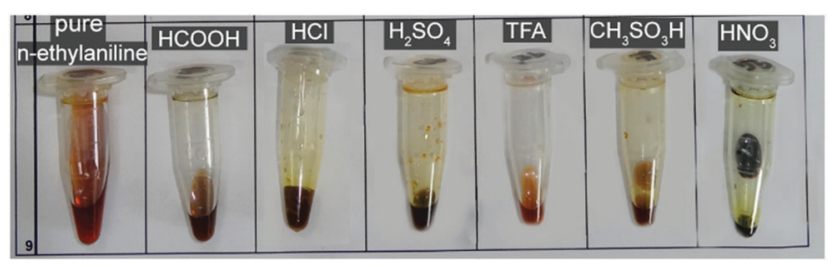

Fig. 1 Visual appearance of pure $n$-ethylaniline and its products as a result of the addition of various acids using the simple Eppendorf tubes highthroughput screen. 
hence formic acid and hydrochloric acid were omitted. The visual appearance of $n$-ethylaniline-acids combinations can be clearly observed in Fig. 1 where the addition of formic acid yielded a dark runny liquid that is not a potential IL. On the other hand, hydrochloric acid produced a solid product when reacted with $n$-ethylaniline. However, sulphuric, trifluoroacetic, and methanesulfonic acids produced relatively viscous liquid products that are potentially ILs. The nitric acid yielded a viscous liquid as well, however, the product was consequently oxidised by the strong acid into a dark black liquid.

The series of 54 aniline derivative-acid combinations that were prepared using the simple Eppendorf tubes high-throughput method, and the 36 samples prepared using the Chemspeed robotic platform were classified according to their visual appearance (i.e. solid or liquid). The results of the reactions carried out by both high-throughput methods are shown in Table 1 according to the visual classification scheme. Some results were different from the simple high-throughput method due to the high accuracy and precise control of the reaction conditions (i.e. greater control over the stoichiometry, and due to maintaining a low temperature during the reaction) by the Chemspeed robotic platform.

The Chemspeed HT method identified five possible PILs, however, we chose $n$-ethylanilinium TFA for the full scale synthesis as it was the least viscous and easiest to handle. The formation of $n$-ethylanilinium TFA PIL was confirmed by the ${ }^{1} \mathrm{H}$ NMR spectra as it shows the proton transfer from the carboxylic group of TFA to the $n$-ethylaniline's nitrogen atom. Fig. 2 shows the absence of the only peak for TFA usually observed at $11.5 \mathrm{ppm}^{20}$ and the appearance of an extra peak at 7.3 ppm (i.e. $n$-ethylaniline region). This peak shift is attributed to the proton transfer between the acid and the $n$-ethylaniline forming a protic ionic liquid.

Furthermore, ${ }^{13} \mathrm{C}$ NMR measurements were carried out to confirm the structure of $n$-ethylaniline and the presence of the TFA in the PIL (Fig. 3). The multiplet peak at $\sim 120 \mathrm{ppm}$ is associated with carbon atoms surrounded by three fluorine atoms $\left(-\mathrm{CF}_{3}\right)$, and the peak at $\sim 160 \mathrm{ppm}$ corresponds to a

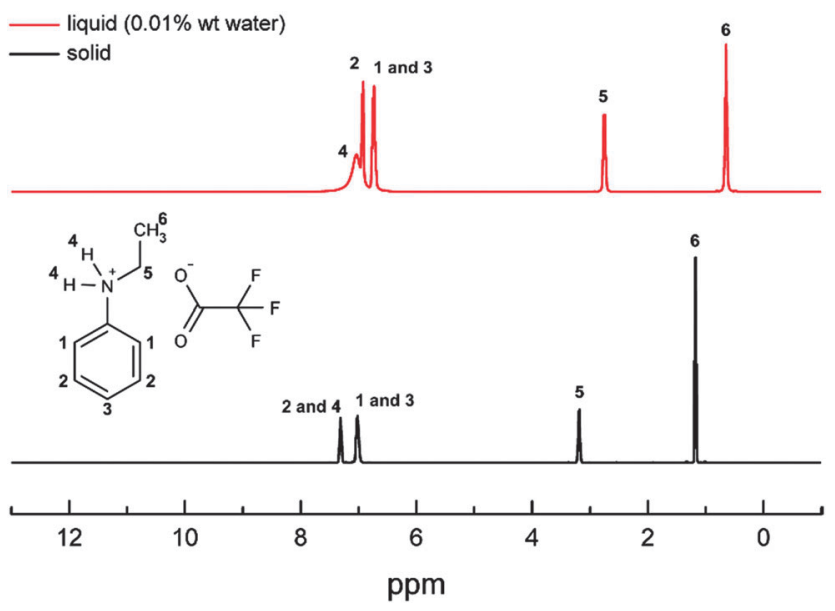

Fig. $2{ }^{1} \mathrm{H}$ NMR spectra of $n$-ethylanilinium TFA.

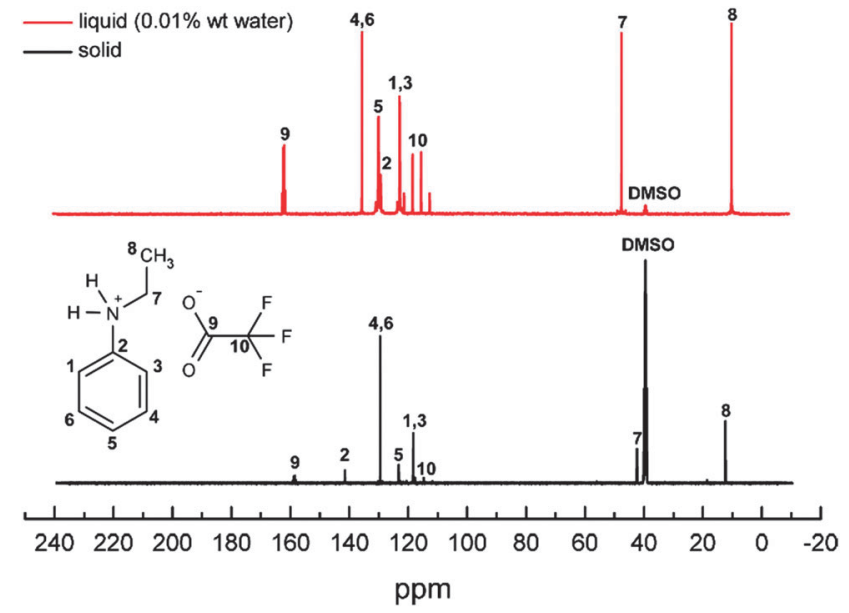

Fig. $3{ }^{13} \mathrm{C}$ NMR spectra of $n$-ethylanilinium TFA.

(-COO-) group which are consistent with the formation of a PIL. Furthermore, the structure of $n$-ethylaniline did not alter upon reaction with TFA as concluded from its characteristic peaks. The combined ${ }^{1} \mathrm{H}$ NMR and ${ }^{13} \mathrm{C}$ NMR data indicate that the reaction of $n$-ethylaniline with TFA took place via proton transfer only, thus forming an ionic compound.

${ }^{1} \mathrm{H}$ NMR and ${ }^{13} \mathrm{C}$ NMR were also used to determine which of the acid-base compounds produced via the Chemspeed robotic platform were ionic liquids (Fig. S1 and S2, ESI $\dagger$ ). The NMR spectra show that $n$-methylaniline yielded ionic liquids with two acids only (i.e. sulphuric acid and trifluoroacetic acid). While $n$-ethylaniline produced three ionic liquids with three acid combinations (i.e. sulphuric acid, trifluoroacetic acid and methanesulfonic acid).

The conductivity of the $n$-ethylanilinium TFA PIL was measured to be $5.2 \mathrm{mS} \mathrm{cm}^{-1}$ at room temperature, compared to $1.0 \mathrm{mS} \mathrm{cm}{ }^{-1}$ for $N$-methylpyrrolidinium TFA and $14.4 \mathrm{mS} \mathrm{cm}^{-1}$ for 2-pyrrolidonium TFA, ${ }^{17,21,22}$ which is consistent with the

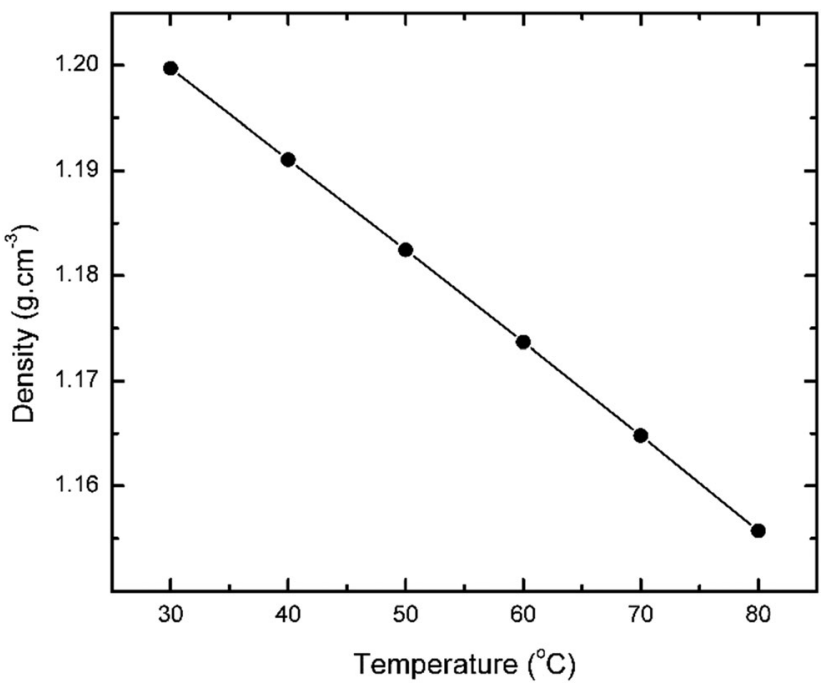

Fig. 4 Graph showing the density of $n$-ethylanilinium TFA as a function of temperature. 


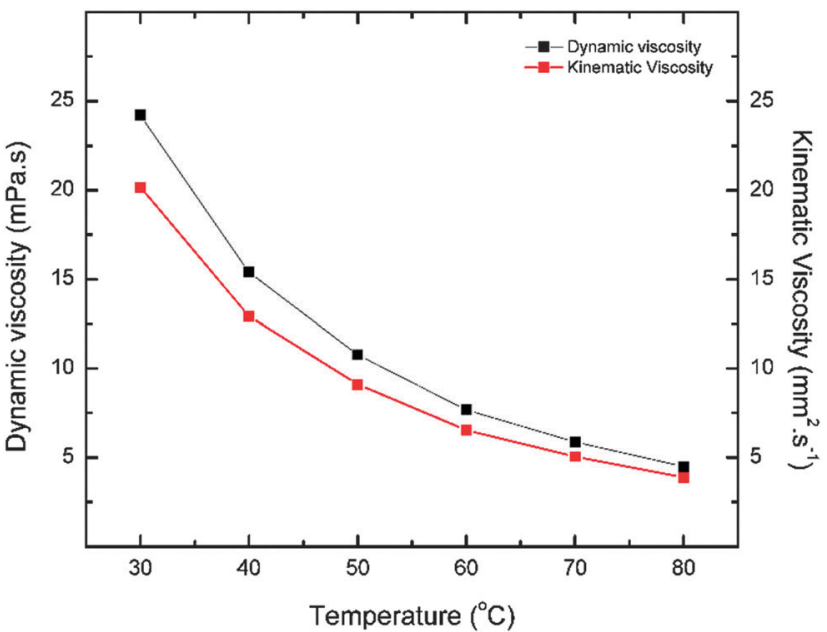

Fig. 5 Graph shows the kinematic and dynamic viscosities of $n$-ethylanilinium TFA as a function of temperature.

formation of an ionic compound. Density measurements were carried out at various temperatures (i.e. 30 to $80{ }^{\circ} \mathrm{C}$, Fig. 4 ). It shows that the $n$-ethylanilinium TFA PIL is approx. $20 \%$ denser than water at near room temperature and its density decreases as a function of temperature.

The dynamic and kinematic viscosities were measured at the same range of temperatures (Fig. 5) to give values of $24.3 \mathrm{mPa} \mathrm{s}^{-1}$ and $20.5 \mathrm{~mm}^{2} \mathrm{~s}^{-1}$ respectively which were much greater than water viscosity values near room temperature. The melting point for both the solid and the liquid form of $n$-ethylanilinium TFA were measured to be approximately $42{ }^{\circ} \mathrm{C}$ and $3{ }^{\circ} \mathrm{C}$ respectively.

\section{Discussion}

The screening process by both the simple Eppendorf tubes and Chemspeed robotic synthesis platform, resulted in five acidbase combinations being identified as potentially useful PILs namely; $n$-methylanilinium sulphate, $n$-methyl-anilinium TFA, $n$-ethylanilinium sulphate, $n$-ethyl-anilinium TFA, and n-ethylanilinium methanesulfonate. Both methods served a complementary role for each other, where the simple Eppendorf tubes high-throughput enabled very rapid screening to narrow down the number of possible acid-base combinations to be tested by the Chemspeed robotic synthesis platform. The Chemspeed high-throughput system then served as an accurate synthesis method.

The Chemspeed high-throughput method revealed that only two aniline derivatives yielded PILs where a hydrocarbon chain is attached to the amine (i.e. $n$-methylaniline and n-ethylaniline). In addition, the longer the hydrocarbon chain is, the less viscous the PIL is as shown in (Fig. S3, ESI $\dagger$ ). This can be explained when the acid reacts with the aniline base to form an anilinium salt, the proton is transferred to the amine group and the nitrogen atom becomes positively charged. With many anilinium molecules in close proximity, they tend to form an offset $\pi$ stacking arrangement where the positively charged nitrogen atoms align on top a)

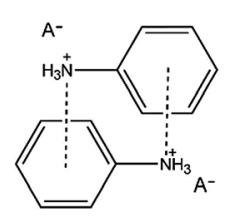

b)

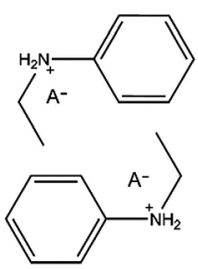

c)

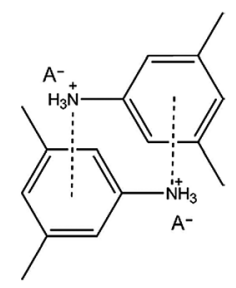

Fig. 6 Illustration showing (a) anilinium salt arranged in an offset $\pi$ stacking configuration, (b) $n$-ethylanilinium salt where the ethyl group is hindering the $\pi$ stacking rearrangement by increasing the proximity between the amine group and the benzene ring, and (c) 3,5-dimethylanilinium salt as an example for the alkyl groups that are attached to the benzene ring and extend on the same plane and do not hinder the $\pi$ stacking rearrangement.

of the $\pi$ electron cloud of the benzene ring (Fig. 6a), hence forming solid salts. However, the hydrocarbon chains act like spacers between the amine group and the benzene ring which reduces the formation of this offset $\pi$ stacking of the anilinium (Fig. 6b), thus preventing the formation of solid salts. It should be noted that 7 out of the 9 studied aniline derivatives formed solids and/or non-ILs liquids (for example 2-ethyl-aniline and 3-ethyl-aniline) as their hydrocarbon chains are attached to the benzene ring instead of the amine group (Fig. 6c). The hydrocarbon chains attached to the benzene ring extend initially on the same plane and hence do not hinder the $\pi$ stacking rearrangement. However, the position of the alkyl group on the benzene ring is shown to affect the state of the product. By comparing the outcome of 2-ethylaniline and 2-ethyl-6-methylaniline, where the ethyl group is on the ortho position, with 3-ethylaniline, where the ethyl group is on the meta position, one can conclude that the derivatives with ortho positioned ethyl groups yielded almost the same results $v$ s. the type of acids (e.g. $\mathrm{H}_{2} \mathrm{SO}_{4} \& \mathrm{TFA} \rightarrow$ solid products $/ \mathrm{CH}_{3} \mathrm{SO}_{3} \mathrm{H} \rightarrow$ liquid products) while meta positioned derivatives resulted in more or less the opposite outcomes with the same acids (e.g. $\mathrm{H}_{2} \mathrm{SO}_{4}$, TFA $\rightarrow$ liquid products $/ \mathrm{CH}_{3} \mathrm{SO}_{3} \mathrm{H} \rightarrow$ solid products). This might be attributed to different steric effects exhibited by the ethyl group on the acids.

The addition of $150 \mu \mathrm{L}(0.015 \mathrm{wt} \%, 162 \mathrm{ppm}$ measured) of water to the product of the full scale synthesis ( $n$-ethylanilinium TFA) in addition to heating to $50{ }^{\circ} \mathrm{C}$ after the purification process were required to convert it back into liquid form. The PIL produced is stable after cooling down to room temperature, hence suggesting that water plays a role in lowering the melting point of the PIL which was measured to be $42{ }^{\circ} \mathrm{C}$ for the solid and $3{ }^{\circ} \mathrm{C}$ after water addition.

In this investigation we screened the stoichiometric acid-base reaction of a broad range of commercially available aniline derivatives with 6 acids. Combinations of these which led to PILs that were liquid at room temperature have the potential to be polymerised into conductive polymers. Of the 54 combinations tested in a HT manner there were five identified as possible candidates for future polymerisation trials, and all of these contained an aniline derivative with a -NHR group. None of the aniline derivatives with $-\mathrm{NH}_{2}$ groups formed PILs which were liquid at room temperature.

Consequently this HT screening approach indicates that future studies should focus on $n$-alkylanilines with different 
alkyl chain lengths (-NHR), extending to a broader range of anilines and acids. While the closely related $n, n$-dialkylanilines $\left(-\mathrm{NR}_{2}\right)$ may form PILs which are liquid at room temperature, they will not be suitable polymerisable candidates due to the absence of protons from the amine groups which are necessary for the polymerisation process to occur. ${ }^{4,5}$

\section{Conclusions}

This is the first high throughput combinatorial approach to anilinium-based PIL design to the best of our knowledge. Compared to the traditional IL synthesis approaches, this is a significantly different and efficient method. Traditionally, IL research has focussed on the rigorous characterisation of a handful of ILs, and there are few studies which use a large number of ILs. ${ }^{16}$ For the aprotic ILs, their synthesis is difficult with many steps and we don't envisage a simple method to high throughput screening. However, due to the simplicity of PIL synthesis, this is a highly viable method to enable the rapid identification of potential PILs, where a select few only then need to be synthesised and characterised in significant quantities. Using high-throughput screening, a wider range of acidbase precursors could be easily trialled to identify potential PILs. Furthermore, the screening process can be designed to identify the PIL with specific properties for specific applications. This new PIL is the first aniline based ionic liquid to the best of our knowledge, which is a very promising reagent as it can play more than one role at once (i.e. solvent, supporting electrolyte and monomer). Initial experiments have been conducted where anilinium-based PILs have been successfully polymerised, and this work will be published in the near future.

\section{Acknowledgements}

The authors acknowledge Dr Shaun C. Howard and Dr Benjamin W. Muir from the Rapid Automated Materials \& Processing centre at CSIRO for providing the technical support and access to the Chemspeed robotic synthesis platform. A.O.M. acknowledges the Australian Research Council for a Future Fellowship (FT110100760). M.E.A. acknowledges CSIRO and RMIT for provision of his PhD studentship. G.A.S. acknowledges the funding from the CSIRO Office of the Chief Executive Julius Career Award.

\section{Notes and references}

1 G. A. Snook, P. Kao and A. S. Best, J. Power Sources, 2011, 196, 1-12.

2 G. Zotti, S. Cattarin and N. Comisso, J. Electroanal. Chem. Interfacial Electrochem., 1988, 239, 387-396.

3 M. E. Abdelhamid, A. P. O'Mullane and G. A. Snook, RSC Adv., 2015, 5, 11611-11626.

4 G. A. Snook, T. L. Greaves and A. S. Best, J. Mater. Chem., 2011, 21, 7622.

5 F. Lux, Polymer, 1994, 35, 2915-2936.

6 G. A. Snook and A. S. Best, J. Mater. Chem., 2009, 19, 4248-4254.

7 T. B. Scheffler and C. L. Hussey, Inorg. Chem., 1984, 23, 1926-1932.

8 M. Galiński, A. Lewandowski and I. Stępniak, Electrochim. Acta, 2006, 51, 5567-5580.

9 J. M. Pringle, O. Ngamna, C. Lynam, G. G. Wallace, M. Forsyth and D. R. MacFarlane, Macromolecules, 2007, 40, 2702-2711.

10 Y. Yang and S. Mu, Electrochim. Acta, 2008, 54, 506-512.

11 D. Wei, C. Kvarnstrom, T. Lindfors and A. Ivaska, Electrochem. Commun., 2007, 9, 206-210.

12 X. Mulet, D. F. Kennedy, C. E. Conn, A. Hawley and C. J. Drummond, Int. J. Pharm., 2010, 395, 290-297.

13 D. Lau, D. G. Hay, M. R. Hill, B. W. Muir, S. A. Furman and D. F. Kennedy, Comb. Chem. High Throughput Screening, 2011, 14, 28-35.

14 R. Cain, S. Narramore, M. McPhillie, K. Simmons and C. W. Fishwick, Bioorg. Chem., 2014, 55, 69-76.

15 W. F. Maier, K. Stowe and S. Sieg, Angew. Chem., Int. Ed., 2007, 46, 6016-6067.

16 T. L. Greaves, K. Ha, B. W. Muir, S. C. Howard, A. Weerawardena, N. Kirby and C. J. Drummond, Phys. Chem. Chem. Phys., 2015, 17, 2357-2365.

17 T. L. Greaves and C. J. Drummond, Chem. Rev., 2008, 108, 206-237. 18 T. L. Greaves, A. Weerawardena, C. Fong, I. Krodkiewska and C. J. Drummond, J. Phys. Chem. B, 2006, 110, 22479-22487.

19 F. Zou, X. Yu, J. Zhang, N. Cheng and X. Huang, Synth. Met., 2015, 204, 76-83.

20 S. Budavari, M. J. O'Neil, A. Smith and P. E. Heckelman, The Merck Index, an Encyclopedia of Chemicals, Drugs, and Biologicals, Merck Co., Inc., Rahway, NJ, 11th edn, 1989.

21 B. Nuthakki, T. L. Greaves, I. Krodkiewska, A. Weerawardena, M. I. Burgar, R. J. Mulder and C. J. Drummond, Aust. J. Chem., 2007, 60, 21-28.

22 M. Yoshizawa, W. Xu and C. A. Angell, J. Am. Chem. Soc., 2003, 125, 15411-15419. 